\title{
Third Ventriculostomy in Late-onset Idiopathic Aqueductal Stenosis Treatment: A Focus on Clinical Presentation and Radiological Diagnosis
}

\author{
Marco LOCATElli, ${ }^{1}$ Riccardo DRAGHI, ${ }^{1,2}$ Andrea Di CRISTOFORI, ${ }^{1,2}$ \\ Giorgio Carrabba, ${ }^{1}$ Mario ZavanOne, ${ }^{1,2}$ Mauro Pluderi, ${ }^{1}$ Diego SPAGnOli, ${ }^{3}$ \\ and Paolo RAMPINI ${ }^{1}$
}

\begin{abstract}
${ }^{1}$ Neurosurgery Unit, Fondazione I.R.C.C.S. "Ca' Granda” Ospedale Maggiore Policlinico, Milan, Italy;

${ }^{2}$ Neurosurgery Unit, Department of Medical and Surgical Pathophysiology of Organs and Transplantation, Università degli Studi di Milano, Milan, Italy;

${ }^{3}$ Neurosurgery Unit, Ospedale Generale "Moriggia-Pelascini”, Gravedona (Como), Italy
\end{abstract}

\begin{abstract}
Endoscopic third ventriculostomy (ETV) is considered the gold standard treatment for obstructive hydrocephalus due to partial or complete obstruction of cerebrospinal fluid (CSF) ventricular pathways caused by mass lesions. However long-term efficacy of this procedure remains controversial as treatment of chronic adult hydrocephalus due to stenosis of Sylvian acqueduct [late-onset idiopathic aqueductal stenosis (LIAS)]. The authors describe clinical presentation, diagnostic investigations in patients affected by LIAS, and define their clinical and radiological outcome after ETV. From January 2003 to December 2008, 13 consecutive LIAS patients treated by ETV were retrospectively reviewed. Pre- and post-operative clinical and radiological findings, including conventional and phase-contrast (PC) cine magnetic resonance imaging (MRI) were investigated. ETV was successfully performed in all patients. Patient's neurological condition improved. No one required a second ETV procedure or shunt implantation. Clinical and radiological results reveal a satisfactory outcome of LIAS patients treated by ETV. At follow-up a clinical improvement could be demonstrated in all cases. Selection criteria of LIAS patients seem to be crucial to obtain satisfactory and long-lasting results. Even in elderly patients with chronic hydrocephalus, ETV can be considered the treatment of choice.
\end{abstract}

Key words: hydrocephalus, late-onset idiopathic aqueductal stenosis, third ventriculostomy, endoscope

\section{Introduction}

Aqueductal stenosis (AS) is a pathological condition causing triventricular obstructive hydrocephalus which requires a clinical and radiological diagnosis. ${ }^{1)}$ The obstruction of cerebrospinal fluid (CSF) pathway may be the result of either spaceoccupying lesions (e.g. posterior fossa or pineal tumors), or primitive stenosis. Patients affected by the last condition develop symptoms like headache, cognitive and gait impairment, and urinary incontinence. ${ }^{1)}$ The presence of such symptoms associated with a primitive CSF obstruction in adult patients is defined as late-onset idiopathic aqueductal stenosis (LIAS). ${ }^{1)}$

Received December 6, 2013; Accepted May 10, 2014
LIAS was first described by Spiller in 1902 and later by other authors, including Dandy in 1920 and Shelden in $1930 .^{2-4)}$

To date endoscopic third ventriculostomy (ETV) is considered the gold standard procedure to treat secondary AS hydrocephalus providing CSF an alternative path to reach the subarachnoid space. ${ }^{5)}$

Nevertheless there are still controversies regarding permanent clinical improvement in the primitive form of AS in adult population. Patients who underwent ETV often experience a temporary clinical improvement, requiring a further positioning of a permanent shunt to control the disease. ${ }^{6)}$

Identifying clinical and radiological criteria of LIAS seems to be crucial in order to determine which patients will respond to ETV. 
In this article the authors reviewed a consecutive series of adult patients affected by hydrocephalus due to primary AS treated with ETV. Particularly, clinical presentation, diagnostic tests, pre-operative, and post-operative radiological findings, and outcomes were analyzed.

\section{Materials and Methods}

Between January 2003 and December 2008, 43 patients underwent ETV at the Neurosurgery Unit of Fondazione I.R.C.C.S “Ca' Granda” Ospedale Maggiore Policlinico, Milan, Italy. Among these, authors selected and retrospectively re-examined adult patients presenting with clinical and radiological diagnosis of hydrocephalus and primitive AS. Clinical diagnosis was assessed taking into account the clinical characterization described by Fukuhara and Luciano in 2001. ${ }^{1)}$ Particularly, in our institution an ETV was proposed to patients if they had symptoms or a clinical history mimicking a progressive decompensation of CSF circulation or an increase of intracranial pressure. Patients with settled symptoms were not proposed for ETV but only for ventriculo-peritoneal shunt.

Radiological inclusion criteria included the absence of CSF flow through the Sylvian aqueduct, measured by phase-contrast (PC) cine magnetic resonance imaging (MRI). Patients who underwent other shunting procedures or were affected by other neurosurgical diseases were not included in the study (Table 1). Written informed consent was obtained before the surgical intervention and the storage of data. Thirteen patients (9 males, 4 females) with a mean age of 56.8 years (22-72 years) at the moment of surgical intervention met selection criteria. The first three patients of the series did not undergo pre-operative PC cine MRI because the MRI program was not available; but they presented morphological findings strongly suggestive of AS at conventional MRI on $\mathrm{T}_{1}$ and $\mathrm{T}_{2}$ weighted images (feature III of Table 1, Fig. 1).

All surgical procedures were performed by a dedicated surgical team. The endoscopic procedure consisted in the coagulation of third ventricle floor between the infundibulum and mammillary bodies and subsequent dilatation by introduction and inflation of a 4-French Fogarty balloon (see technical note below). All procedures were recorded on VHS tapes or DVD and then critically reviewed. Three months after surgery all patients were evaluated by clinical examination. Then, they were evaluated once a year with a clinical examination and a PC cine MRI to document the patency of the ventriculostomy.
In order to evaluate the overall outcome, the following data were considered: pre-operative neurological and instrumental findings, the restoration of back-and-ward movement of the third ventricle floor after shunt creation, post-operative neurological examination, and MRI findings. Patients' followup ranged from 3 months to 52 months (average follow-up $=30.6$ months).

Table 1 Late-onset idiopathic aqueductal stenosisinclusion criteria

I. Age $>18$ years

II. Radiological findings on conventional magnetic resonance imaging (MRI):

- supratentorial ventricular dilatation;

- comparatively small fourth ventricle;

III. Radiological findings:

cerebrospinal flow absence at the aqueduct documented by phase-contrast MRI and/or

obvious supratentorial ventriculomegaly associated with downward bulging of the third ventricle

floor and/or cerebrospinal fluid (CSF) pathway obstruction at the aqueduct visible on $\mathrm{T}_{1}$ and $\mathrm{T}_{2}$ sagittal sequences

IV. Suspected hydrocephalus neurological condition

V. No previous shunting procedure

VI. No other neurosurgical disease associated with Late-onset Idiopathic aqueductal stenosis

VII. Endoscopic third ventriculostomy performed from 2003 to 2008

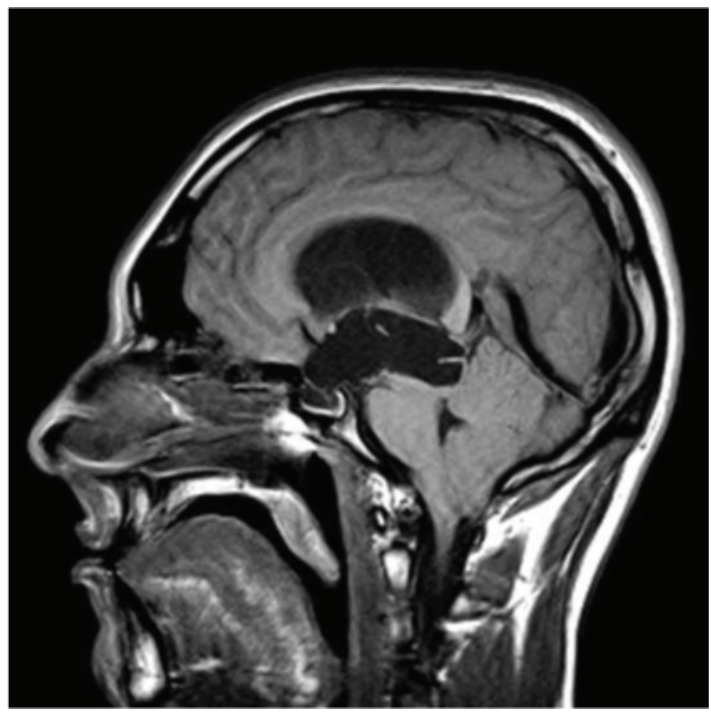

Fig. $1 T_{1}$ MRI showing huge supratentorial ventricular dilatation. An evident obstruction of proximal Sylvius aqueduct suggested aqueductal stenosis diagnosis. MRI: magnetic resonance imaging. 


\section{ETV surgical technique}

The patients' heads were positioned in a neutral position, immobilized by Mayfield clamp and then flexed $30^{\circ}$ in order to reduce excessive CSF loss and subsequent pneumocephalus.

A right pre-coronal burr hole $(14 \mathrm{~mm} \varnothing) 2.5 \mathrm{~cm}$ lateral to midline was performed. Dura mater was opened by a cross-like incision and its edges were coagulated. Endoscope insertion trajectory was previously planned with neuronavigation workstation (Radionics, Burlington, Massachusetts, USABrainLAB Inc. Westchester, Illinois, USA). We chose the most direct trajectory to third ventricle floor allowing reduced tractions on the fornix during ventriculostomy creation. A 14-French Cushing gauge was used to cannulate the lateral ventricle. Once the correct position was verified by removing the stylet, an endoscope with a working channel $\left(0^{\circ}\right.$ lens-4.2 outer diameter, Aesculap-B. Braun Melsungen AG, Tuttlingen, Germany / Karl Storz GmbH \& Co. KG, Tuttlingen, Germany) was introduced. Anatomic landmarks were identified. The CSF ventricular pathway was navigated through Monroe's foramen and the floor of the third ventricle was reached by following the fornix. Superficial coagulation of the floor of the third ventricle was performed between the mammillary bodies and the infundibulum and the coagulated area was gently perforated by a 1-mm blunt probe. The ventriculostomy was dilated by repeated inflation of a 4-French Fogarty balloon. The endoscope was then pushed forward into the interpeduncular cystern and any residual of Liliequist's membrane located directly below the stoma were gently ablated, in order to avoid potential CSF obstruction to vault subarachnoid spaces.

When back-and-forward movements of the third ventricle floor after the shunt creation were observed, this was recorded in the operative report. At the end of the procedure the endoscope was retracted. The craniectomy was closed with Gelfoam (Baxter Healthcare Corporation, Hayward, California, USA). Galea and skin were sutured. All the procedures were recorded on VHS tapes or DVD and then reviewed.

\section{Results}

\section{Clinical onset}

Presenting clinical conditions (i.e., the reason why the patient presented to clinical attention) were variable according to age. Gait disorder was the most frequent onset sign and symptom (8 patients, $62 \%$ ). All patients presenting with gait ataxia were older than 50, with a median age of 67 years (average age $=65.8$ years). Gait disorder included postural instability, unsteadiness, broad-based gait with short stride length, resembling ataxia of normal pressure hydrocephalus (NPH). Headache was present in two patients $(15 \%)$. One of them described long-standing episodic headache that became permanent over the last months and was associated with drop-attacks; the other one complained acute pulsating headache that started only 4 days before admission to the emergency room. These patients were 25 and 32 years old, respectively.

Two patients came to our attention for progressive cognitive deterioration, characterized by short-term memory and concentration impairment; they were aged 65 and 69. One patient required medical aid for visual field deficits: conventional MRI showed a wide downward bulging of the third ventricle floor, exerting direct compression on the optic chiasm (22 years old).

According to the classification proposed by Fukuhara and Luciano, patients were divided into three groups according to the time of onset of clinical symptoms (see Table 2).

\section{Pre-operative radiological findings}

All patients underwent pre-operative conventional MRI; in 10 patients PC imaging was also performed.

Table 2 Patients features

\begin{tabular}{ccccc}
\hline Case & $\begin{array}{c}\text { Age } \\
\text { (years), } \\
\text { Sex }\end{array}$ & $\begin{array}{c}\text { Fukuhara } \\
\text { class }\end{array}$ & $\begin{array}{c}\text { Onset } \\
\text { symptoms }\end{array}$ & $\begin{array}{c}\text { Phase-contrast } \\
\text { cine } \\
\text { MRI finding }\end{array}$ \\
\hline 1 & $68, \mathrm{~F}$ & III & Gait & Not done \\
2 & $32, \mathrm{M}$ & I & Headache & Not done \\
3 & $69, \mathrm{M}$ & III & Cognitive & Not done \\
4 & $22, \mathrm{M}$ & II & Visual deficit & + \\
5 & $65, \mathrm{M}$ & II & Cognitive & + \\
6 & $63, \mathrm{~F}$ & III & Gait & + \\
7 & $72, \mathrm{~F}$ & III & Gait & + \\
8 & $25, \mathrm{M}$ & II & Headache & + \\
9 & $71, \mathrm{M}$ & II & Gait & + \\
10 & $52, \mathrm{M}$ & III & Gait & + \\
11 & $69, \mathrm{M}$ & III & Gait & + \\
12 & $65, \mathrm{~F}$ & III & Gait & + \\
13 & $66, \mathrm{M}$ & III & Gait & + \\
\hline
\end{tabular}

LIAS patients' series. According to Fukuhara and Luciano (2001), patients were divided in three classes according to hydrocephalus duration. Class I (acute): symptoms lasting less than 1 month. Class II (subacute): 1 month $<,<6$ months. Class III (chronic): more than 6 months. +: no flow through aqueduct of Sylvius. Not done: patients underwent a brain MRI with sagittal $\mathrm{T}_{1}$ and $\mathrm{T}_{2}$ weighted images which documented a stenosis of the aqueduct of Sylvius, LIAS: late-onset idiopathic aqueductal stenosis, MRI: magnetic resonance imaging. 
All conventional MRI sequences revealed the morphological alterations reported in Table 1, point II. One patient showed a strongly pronounced downward bulging of third ventricle floor, imprinting the optic chiasm. PC cine MRI data confirmed highly absent flow through the Sylvian aqueduct in all patients with morphological aspect of severe aqueductal stenosis.

In two patients, although the aqueduct appeared only slightly restricted on the conventional images, PC sequences allowed us to document a functional AS (Fig. 2).

\section{Intraoperative findings}

The ETV was performed successfully in all patients. The restoration of back-and-ward movements of third ventricle floor was observed in all cases. In the first two cases a catheter connected to a subcutaneous Ommaya reservoir was implanted to control intracranial hypertension in case of ETV failure.

No major complications were recorded; one patient experienced moderate bleeding from the stoma
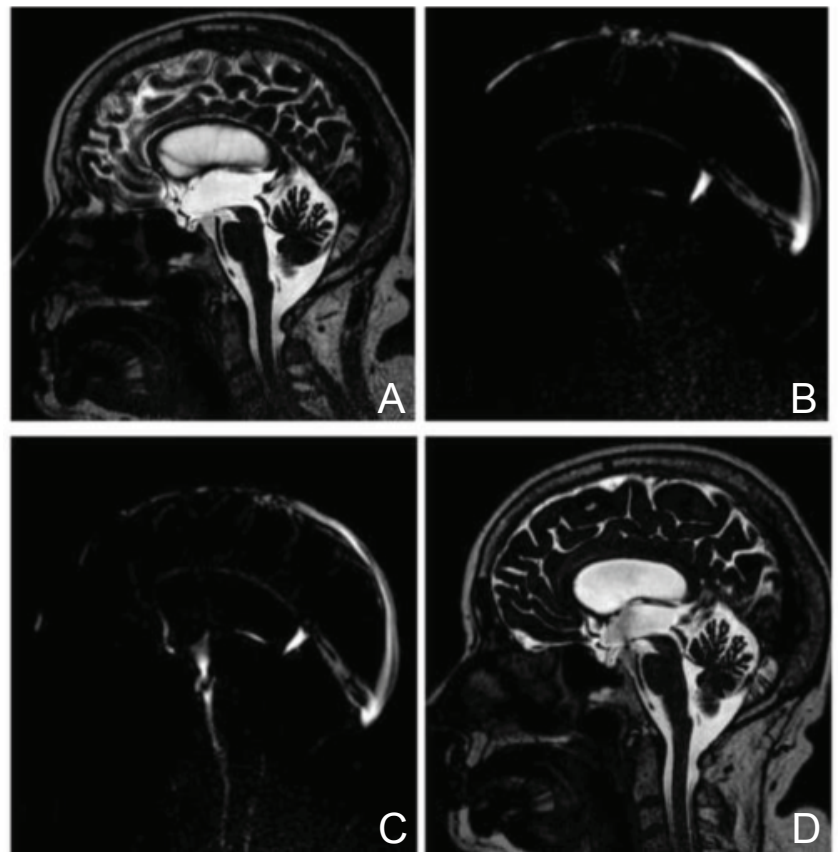

Fig. 2 A: CISS MRI showing supratentorial ventricular dilatation and the anatomical detail of the aqueduct. A thin sepimento appears before the fourth ventricle inlet. B: Pre-operative PC cine MRI documents the absence of CSF flow through the aqueduct. C: One-year postoperative PC cine MRI documenting CSF flow through the stoma. D: One-year post-operative CISS MRI showing reduction of supratentorial ventricles size; the proximal part of aqueduct is clearly less dilated. Flow-void sign through ostomy can be appreciated. CISS: constructive interference in steady state, CSF: cerebrospinal fluid, MRI: magnetic resonance imaging. walls which was well controlled by inflation of a Fogarty balloon.

\section{Clinical outcome}

No CNS or wound infections were documented in the post-operative period. Patients were evaluated after an average follow-up period of 30.6 (3-52) months from surgery. The follow-up data for each patient are reported in Table 3. Outcome data was analyzed according to the preoperative classes (Table 4). Unfortunately, the only patient with acute onset LIAS was lost at 3 months follow-up; this patient, who presented pulsating headache and bilateral papilloedema at onset, reported the complete resolution of cephalea and examination of fundus oculi revealed a normal optic papilla seven days after surgery. He performed a 3-month PC-MRI which demonstrated the persistence of CSF flow through the stoma.

Among the group of subacute LIAS (four patients), all patients showed improvement in gait; one patient revealed complete resolution of walking disorders. Two patients complained long-standing episodic headache that improved after ETV. Urinary incontinence with urgency and occasional incontinence was reported by three patients; two of whom reported improvement of urinary disorder, while one patient remained stable. No cognitive improvement was recorded in this class. Among the eight patients included in the chronic LIAS group, six patients presented gait instability at the time of surgery; in three of them gait disorder resolved completely, while an improvement was observed in the remaining three patients. Urinary incontinence was recorded in four patients; this disorder improved in two of them. Cognitive evaluation improved in four of the six patients presenting with cognitive deterioration, while the other two showed no variation. One patient returned to his previous professional and social activities.

\section{Post-operative radiological findings}

All patients underwent post-operative PC cine MRI; long-term control demonstrated patency of the ventriculostomy in all cases. CSF flow values through aqueduct were stable in all cases.

\section{Discussion}

LIAS is a primitive obstructive hydrocephalus due to a stenosis of the acqueduct of Sylvius. Its symptoms develop in adulthood probably for an impairment of the CSF circulation in a chronic compensated hydrocephalus. ${ }^{7}$ Patients affected by LIAS can be divided into two groups: one with symptoms related to increased intracranial hypertension and one mimicking 
Table 3 Our series of patients affected by LIAS

\begin{tabular}{|c|c|c|c|c|c|}
\hline $\mathrm{Pt}$ & $\begin{array}{l}\text { Age (years), } \\
\quad \text { Sex }\end{array}$ & $\begin{array}{l}\text { Fukuhara } \\
\text { class }\end{array}$ & Pre-operative symptoms & Post-operative symptoms & $\begin{array}{l}\text { Follow-up } \\
\text { (months) }\end{array}$ \\
\hline 1 & $68, \mathrm{~F}$ & III & $\begin{array}{l}\text { Gait impairment and instability and } \\
\text { urinary incontinence }\end{array}$ & $\begin{array}{l}\text { Improvement of gait instability and } \\
\text { urinary incontinence }\end{array}$ & 52 \\
\hline 2 & $32, \mathrm{M}$ & I & $\begin{array}{l}\text { Acute and intense headache for } \\
4 \text { days and bilateral papilledema }\end{array}$ & $\begin{array}{l}\text { Improvement of headache and } \\
\text { papilloedema }\end{array}$ & 3 \\
\hline 3 & $69, \mathrm{M}$ & III & $\begin{array}{l}\text { Subjective dizziness or instability for } \\
1 \text { year, mild cognitive impairment } \\
\text { and persistent headache for } 2 \text { years }\end{array}$ & $\begin{array}{l}\text { Improvement of headache and } \\
\text { cognitive impairment. Resolution of } \\
\text { subjective dizziness or instability }\end{array}$ & 16 \\
\hline 4 & $22, \mathrm{M}$ & II & $\begin{array}{l}\text { Headache and bilateral hypovisus } \\
\text { with papilloedema }\end{array}$ & $\begin{array}{l}\text { Improvement of headache, visus and } \\
\text { papilloedema }\end{array}$ & 34 \\
\hline 5 & $65, \mathrm{M}$ & II & $\begin{array}{l}\text { Early dementia and gait impairment } \\
\text { for } 6 \text { months. Urinary incontinence. } \\
\text { Papilloedema }\end{array}$ & $\begin{array}{c}\text { Improvement of gait impairment and } \\
\text { cognitive impairment. Persistence } \\
\text { of urinary incontinence. No } \\
\text { papilloedema }\end{array}$ & 50 \\
\hline 6 & $63, \mathrm{~F}$ & III & Ataxic gait with frequent falls & Resolution of gait ataxia & 51 \\
\hline 7 & $72, \mathrm{~F}$ & III & $\begin{array}{l}\text { Gait impairment, mild cognitive } \\
\text { impairment, and urinary } \\
\text { incontinence }\end{array}$ & $\begin{array}{l}\text { Improvement of gait and cognitive } \\
\text { impairment. Persistence of urinary } \\
\text { incontinence }\end{array}$ & 30 \\
\hline 8 & $25, \mathrm{M}$ & II & $\begin{array}{l}\text { Persistent headache for } 3 \text { months, gait } \\
\text { instability, and urinary incontinence. } \\
\text { Episodic drop-attacks. Papilloedema }\end{array}$ & $\begin{array}{l}\text { Improvement of headache. Resolution } \\
\text { of gait impairment and papilloedema. } \\
\text { Improvement of urinary incontinence }\end{array}$ & 50 \\
\hline 9 & $71, \mathrm{M}$ & II & $\begin{array}{l}\text { Ataxic gait and episodic urinary } \\
\text { incontinence for } 6 \text { months }\end{array}$ & $\begin{array}{c}\text { Resolution of gait impairment and } \\
\text { urinary incontinence }\end{array}$ & 40 \\
\hline 10 & $52, \mathrm{M}$ & III & $\begin{array}{l}\text { Gait impairment for } 1 \text { year, mild } \\
\text { cognitive impairment, and episodic } \\
\text { urinary incontinence }\end{array}$ & $\begin{array}{c}\text { Resolution of gait and urinary } \\
\text { impairment. Stability of cognitive } \\
\text { impairment }\end{array}$ & 28 \\
\hline 11 & $69, \mathrm{M}$ & III & $\begin{array}{l}\text { Progressive cognitive impairment and } \\
\text { subjective dizziness and instability }\end{array}$ & $\begin{array}{l}\text { Resolution of cognitive impairment } \\
\text { and improvement of subjective } \\
\text { instability }\end{array}$ & 20 \\
\hline 12 & $65, \mathrm{~F}$ & III & $\begin{array}{l}\text { Persistent morning headaches for } 2 \\
\text { years, gait instability for } 5 \text { months, } \\
\text { and progressive cognitive impairment }\end{array}$ & $\begin{array}{l}\text { Resolution of gait instability, } \\
\text { resolution of cognitive impairment, } \\
\text { decrease of morning headaches }\end{array}$ & 7 \\
\hline 13 & $66, \mathrm{M}$ & III & $\begin{array}{l}\text { Gait impairment for } 1 \text { year, mild } \\
\text { cognitive impairment, and urinary } \\
\text { incontinence for } 7 \text { months }\end{array}$ & $\begin{array}{l}\text { Improvement of gait impairment. } \\
\text { Stability of cognitive impairment. } \\
\text { Persistence of urinary incontinence }\end{array}$ & 17 \\
\hline
\end{tabular}

According to Fukuhara and Luciano (2001), patients were divided in three classes according to hydrocephalus duration. Class I (acute): symptoms lasting less than 1 month. Class II (subacute): 1 month $<,<6$ months. Class III (chronic): more than 6 months, LIAS: late-onset idiopathic aqueductal stenosis.

Table 4 Long-term outcome according to Fukuhara classes

\begin{tabular}{lcccc}
\hline Class (n ${ }^{\circ}$ of patients) & Gait & Headache & Cognitive impairment & Urinary incontinence \\
\hline I (1) & $/$ & $1(++)$ & $/$ & $/$ \\
II (4) & $4(1++; 3+)$ & $2(+)$ & $2(1+; 1=)$ & $3(2+; 1-)$ \\
III (8) & $6(3++; 3+)$ & $2(+)$ & $6(2++; 2+, 2=)$ & $4(2+; 2=)$ \\
\hline
\end{tabular}

Long-term results after ETV are presented according to Fukuhara's classification and individual pre-operative signs/symptoms at the end of the follow-up. ++: resolved, +: improved, =: stable, -: worsen, ETV: endoscopic third ventriculostomy. 
NPH Hakim's triad. ${ }^{1)}$ Particularly, LIAS differs from a secondary obstructive hydrocephalus which is characterized by symptoms related to intracranial hypertension and/or acute obstruction to the CSF circulation (e.g., acute cephalea, nausea, vomiting, and coma). Clinical features of LIAS were first described in a pre-MRI era and more recently by Fukuhara in 2001.,8-11)

Etiology remains uncertain. In the middle of the 20th century, an anatomical classification was proposed, based on the appearance of the Sylvian aqueduct. It was assumed that the pathophysiological mechanism of the disease would be malformative or inflammatory, the latter due to an abnormal proliferation of subependymal glia of the Sylvius aqueduct. ${ }^{3,11)}$

Although endocrine dysfunction in LIAS was previously reported in literature, neurologic symptoms remain the principal clinical presentation in these patients. ${ }^{9,10,12)}$

The neurological clinical status of LIAS at onset can be divided in two categories: (1) patients with intracranial hypertension syndrome and (2) patients experiencing a clinical condition similar to Hakim's symptom triad. These two categories were first described by Fukuhara and Luciano. Particularly, older age at the time of clinical onset was stressed as an influencing factor on chronic form of this disease. ${ }^{1)}$

In this series three different groups of patients are described in relation with disease duration (acute, subacute, and chronic LIAS).

In the acute group only one patient (a 32-year-old male) was included, with symptoms lasting for less than a month. Neurological examination revealed an intense pulsating headache and bilateral papilloedema. Because of the ophthalmological findings this patient underwent an urgent ETV, with rapid improvement of clinical conditions.

The subacute LIAS group included four patients. All patients complained gait disturbance with instability; moreover, two suffered urinary urgency with occasional incontinence and cognitive deficit. Importantly, in this category papilloedema was detected in two patients.

The chronic LIAS group included eight patients. Seven subjects presented with gait impairment, most of whom also suffered from cognitive deterioration.

As in previous reports, in our series the subacute/chronic categories of LIAS were the most represented. ${ }^{1)}$

Though cases of acute LIAS are rare, the importance of a correct and prompt diagnosis should be underlined as neurosurgical treatment (ETV) is mandatory to prevent morbidity and mortality.

Moreover, we suggest timely surgical treatment also in subacute LIAS, as these patients often show optic disc alterations at presentation.

ETV is considered at present the gold standard to treat hydrocephalus due to secondary aqueduct stenosis as the stoma provides CSF a sort of bypass to reach the subarachnoid space. ${ }^{5)}$

The rate of success of ETV in primitive forms of aqueduct stenosis remains undetermined, especially in adult patients affected with LIAS. In fact, patients submitted to ETV often show only temporary clinical improvement, making the positioning of a ventricularperitoneal shunt necessary to control the disease progression. ${ }^{6)}$ It is unnecessary to underline the disadvantages of shunt procedures in comparison to endoscopic surgery.

Therefore, the identification of clinical and radiological criteria can be crucial to predict the success of ETV.

In our series all patients improved after treatment by ETV, with a lasting amelioration of at least one of the pre-operative disorders being obtained in all cases. At the last follow-up visit their clinical condition can be considered satisfactory. The improvement of clinical symptoms, in our opinion, is related to the clinical history of our group of patients which resembles a progressive decompensation of CSF circulation commonly observed in chronic obstructive hydrocephalus. In fact, among our series, all the three different classes of patients show a clinical history characterized by a progressive impairment of the CSF circulation which is related to the obstruction of the acqueduct of Silvio. This is probably the reason why all patients had an improvement after surgery and why none of them underwent to a ventriculo-peritoneal shunt or a second ETV.

The most frequent clinical presentation of LIAS was related with prolonged period of increased intracranial pressure (subacute/chronic LIAS).

These LIAS patients have clinical features which are substantially indistinguishable from those of patients affected with NPH. Furthermore, it is not clear whether and when LIAS patients can "crash" into an acute intracranial hypertension syndrome. This doubt is the key point to consider before deciding to perform an ETV procedure as soon as the correct diagnosis of LIAS is made.

Moreover, while ETV in obstructive hydrocephalus is known to be associated with a high-rate of good long-term outcome, NPH ventriculostomy presents controversial results and ventricular derivation remains nowadays the standard treatment in most neurosurgical centers. ${ }^{13-15)}$ Hence, the importance of distinguishing these two conditions is mandatory.

Brain MRI is the gold standard radiological exam to make differential diagnosis between these two 
diseases. MRI images in NPH show panventriculomegaly with cortical gyri compressed versus the cranium vault. As for LIAS, MRI images are typically characterized by supratentorial ventricular enlargement (in comparison to fourth ventricle), associated with morphologic evidence of restriction or obstruction of Sylvius aqueduct. The alteration of the aqueduct, documented by thin layer $\mathrm{T}_{2}$ sagittal sequences, can show abnormalities such as those proposed by Russel. These features can suggest LIAS diagnosis, but they do not allow to determine their role in influencing CSF flow.

Nevertheless, the distinction between these two radiological patterns can be difficult; it is not infrequent to observe minimal differences in size between supratentorial and fourth ventricles. Futhermore, in these cases a patent aqueduct on radiological examinations could be insufficient to exclude LIAS. ${ }^{15)}$ Interestingly, Stoquart-El Sankari et al. described seven cases of hydrocephalus with comparative small fourth ventricle in relation to the dilated supratentorial ventricles without any other direct or indirect sign of aqueduct obstruction on conventional MRI. In contrast, in these patients there was evidence of no CSF flow at PC-MR sequences. Five patients of this group underwent ETV, with complete recovery of clinical symptoms. ${ }^{16)}$

PC cine MRI findings in NPH and AS are different. While in AS this technique reveals no CSF flow through the aqueduct, NPH it shows higher peak systolic and diastolic velocities and a reduced stroke volume (negative in some cases), due to the strongly reduced compliance of the intracranial system in combination with a patent aqueduct. ${ }^{17,18)}$

It could be concluded that PC-MRI could be considered an essential instrument for the diagnosis of LIAS, as it offers a non invasive exam to measure CSF flow through the aqueduct. In this series, 10 patients with suspected hydrocephalus from idiopathic AS underwent PC cine MRI. This technique confirmed the hypothesis of a highly reduced or absent CSF flow through Sylvian aqueduct which was previously determined by plain morphologic features of conventional MRI. PC cine MRI was positive for LIAS also in the two cases with no evident aqueductal stenosis. Patients underwent ETV considering the results of PC cine MRI. During the procedure, active pulse of the third ventricle floor after ventriculostomy was documented and, more importantly, a stable improvement of preoperative clinical conditions was obtained with a mean follow-up of 30.6 months.

Our results are encouraging, suggesting that an improvement after ETV can be achieved with a strict clinical evaluation indicative of a progressive impairment of CSF circulation associated with the presence of an acqueductal stenosis. The crucial technique for a correct radiological diagnosis was PC cine MRI, which provided measurement of CSF flow through the aqueduct. As a matter of fact, when one of the two conditions is absent, only a ventriculo-peritoneal shunt is proposed. Finally, we aim to improve our clinical assessment with a neuropsychological examination before and after ETV, particularly in patients with subacute and chronic LIAS.

\section{Conclusion}

The results presented in this article revealed a good outcome of LIAS patients treated by ETV with a mean follow-up duration of 30.6 months. LIAS diagnosis was confirmed by PC cine MRI. The retrospective analysis together with the low number of patients represent the limits of this study and do not allow statistical evaluation. It should be considered that LIAS belongs to rare diseases. Nevertheless, the low incidence of this condition and the accurate selection of cases in the present series strengthen our results. In the suspect of LIAS at neurological examination and conventional MRI, most patients underwent PC cine MRI to confirm the diagnosis. If this exam revealed an absent flow through the Sylvian aqueduct, ETV procedure was performed obtaining a restoration of back-and-ward movement of the third ventricle floor. Adequate flow through the ventriculostomy during the surgical procedure and its persistency was demonstrated by post op PC cine MRI. At follow-up all patients showed a permanent neurological improvement.

In the authors' opinion, PC cine MRI is an invaluable instrument for diagnosis, providing objective data on flow through Sylvian aqueduct. On the other hand, a clinical history suggesting a progressive decompensation of CSF circulation seem to be the best predicting factor to assess the outcome of patients.

Future efforts will be directed to prospectively determine the outcome of LIAS patients, exploring not only classical neurologic features but also neuropsychological aspects before and after treatment.

\section{Conflicts of Interest Disclosure}

The authors have no conflicts of interest.

\section{References}

1) Fukuhara T, Luciano MG: Clinical features of lateonset idiopathic aqueductal stenosis. Surg Neurol 
55: 132-136; discussion 136-137, 2001

2) Spiller WG: Two cases of partial internal hydrocephalus from closure of the interventricular passages: with remarks on bilateral contractures caused by a unilateral cerebral lesion. Am J Med Sci; 124: 144-55, 1902

3) Dandy WE: The diagnosis and treatment of hydrocephalus resulting from strictures of the aqueduct of sylvius. Surg Gynecol Obstet 31: 340-358, 1920

4) Shelden WD, Parker HL, Kernohan JW: Occlusion of the aqueduct of sylvius. Arch Neurol Psych 23: 1183-1202, 1930

5) Jones RF, Stening WA, Brydon M: Endoscopic third ventriculostomy. Neurosurgery 26: 86-92, 1990

6) Tisell LE, Almstrom O, Stephensen H, Tullberg M, Wikkelsö C: How effective is endoscopic third ventriculostomy in treating adult hydrocephalus caused by primary aqueductal stenosis? Neurosurgery 46(1): 104-110, 2000

7) Edwards RJ, Dombrowski SM, Luciano MG, Pople1 IK: Chronic hydrocephalus in adults. Brain Pathol 14: 325-336, 2004

8) Harrison MJG, Robert CM, Uttley D: Benign aqueduct stenosis in adults. J Neurol Neurosurg Psychiatry 37: 1322-1328, 1974

9) Little JR, Houser OW, MacCarty CS: Clinical manifestations of aqueductal stenosis in adults. J Neurosurg 43(5): 546-552, 1975

10) Nag TK, Falconer MA: Non-tumoral stenosis of the aqueduct in adults. Br Med J 2: 1168-1170, 1966

11) Russell DS: Spec. Rep. Ser. med. Res. Counc., no 265, London, HMSO, 1949.

12) Wilkinson HA, LeMay M, Drew JH: Adult aqueductal stenosis. Arch Neurol 15: 643-648, 1966

13) Gangemi M, Mascari C, Maiuri F, Godano U, Donati P, Longatti PL: Long-term outcome of endoscopic third ventriculostomy in obstructive hydrocephalus. Minim Invasive Neurosurg 50: 265-269, 2007

14) Kandrian D, van Gelder J, Florida D, Jones R, Vonau M, Teo C Stening W, Kwok B: Long-term reliability of endoscopic third ventriculostomy. Neurosurgery 56(6): 1271-1278; discussion 1278, 2005

15) Gangemi M, Maiuri F, Naddeo M, Godano U, Mascari C, Broggi G, Ferroli P: Endoscopic third ventriculostomy in idiopathic normal pressure hydrocephalus: an Italian multicenter study. Neurosurgery 63(1): 62-67; discussion 67-69, 2008

16) Stoquart-El Sankari S, Lehmann P, Gondry-Jouet C, Fichten A, Godefroy O, Meyer ME, Baledent O: Phase-contrast MR imaging support for the diagnosis of aqueductal stenosis. AJNR Am J Neuroradiol 30(1): 209-214, 2009

17) Battal B, Kocaoglu M, Bulakbasi N, Husmen G, Tuba Sanal H, Tayfun C: Cerebrospinal fluid flow imaging by using phase-contrast MR technique. $\mathrm{Br} J$ Radiol 84: 758-765, 2011

18) Kim DS, Choi JU, Huh R, Yun PH, Kim DI: Quantitative assessment of cerebrospinal fluid hydrodynamics using a phase-contrast cine MR image in hydrocephalus. Childs Nerv Syst 15: 461-467, 1999

Address reprint requests to: Andrea Di Cristofori, MD, Neurosurgery Unit, Department of Medical and Surgical Pathophysiology of Organs and Transplantation, Università degli Studi di Milano, Milan, Italy-Fondazione I.R.C.C.S. "Ca’ Granda” Ospedale Maggiore Policinico, Via Francesco Sforza 35 (Padiglione Monteggia), Milano 20122, Italy. e-mail: andrea.dicristofori@gmail.com 